\title{
Predictors of long-term renal function after kidney surgery for patients with preoperative chronic kidney disease
}

Andrew Silagy ${ }^{1,2}$; Emily Zabor ${ }^{3}$; Roy Mano ${ }^{1}$; Renzo DiNatale ${ }^{1}$; Julian Marcon ${ }^{1}$; Mahyar

Kashani $^{1}$; Kyle Blum ${ }^{1}$; Eduard Reznik ${ }^{4}$; Edgar Jaimes ${ }^{5}$; Jonathan Coleman ${ }^{1}$; A. Ari Hakimi ${ }^{1}$;

Paul Russo ${ }^{1}$

${ }^{1}$ Urology Service, Department of Surgery, Memorial Sloan Kettering Cancer Center, New York, NY, United States;

${ }^{2}$ Department of Surgery, University of Melbourne, Austin Hospital, Melbourne, Australia; ${ }^{3}$ Department of Epidemiology and Biostatistics, Memorial Sloan Kettering Cancer Center, New York, NY, United States; ${ }^{4}$ Center for Molecular Oncology, Memorial Sloan Kettering Cancer Center, New York, NY, United States; ${ }^{5}$ Renal Service, Memorial Sloan Kettering Cancer Center, New York, NY, United States

Acknowledgement: This study was supported by the National Cancer Institute.

Cite as: Can Urol Assoc J 2020 July 27; Epub ahead of print. http://dx.doi.org/10.5489/cuaj.6485

Published online July 27, 2020

$* * *$

\section{Abstract}

Introduction: We evaluated the trajectory of estimated glomerular filtration rate (eGFR) after kidney surgery in patients with kidney cancer and chronic kidney disease (CKD).

Methods: We identified 1204 consecutive patients in our institutional database with preoperative CKD undergoing partial or radical nephrectomy from 1998-2016. Postoperative eGFR was tracked, with patients censored when receiving dialysis or kidney transplantation. A multivariable mixed-effects models assessed associations between preoperative baseline patient and tumor characteristics, and longitudinal eGFR. The Kaplan-Meier method and multivariable Cox regression were used to estimate overall survival, cancer-specific survival, and cumulative incidence of dialysis.

Results: Preoperatively, 892 (74.1\%), 271 (22.5\%), and 41 (3.4\%) patients had CKD stage 3a, $3 \mathrm{~b}$, and $4 / 5$, respectively. There were 55 patients dialyzed and 355 deaths ( 99 from kidney cancer). Median followup was 8.1 years, with 25781 postoperative eGFR measurements. Factors associated with decreasing eGFR postoperatively included radical nephrectomy, male gender, older age, increased body mass index (BMI), and cardiovascular risk factors. We observed a significant interaction effect between time from surgery and preoperative CKD stage: the eGFR of stage 3 a patients improved, while stage $\geq 3 \mathrm{~b}$ declined $(\mathrm{p}<0.001)$. The two- year and five-year cumulative incidence of dialysis was $1.8 \%(1.1-2.6 \%)$ and $3.1 \%(2.2-4.2 \%)$, respectively. The 
cumulative incidence of dialysis, with death as a competing event, significantly differed by preoperative CKD stage.

Conclusions: Preoperative CKD stage $\geq 3 \mathrm{~b}$ is independently associated with a higher risk of declining renal function, dialysis, and mortality. With careful selection, patients with preoperative CKD withstand kidney surgery with low rates of dialysis.

\section{Introduction}

Surgery is a key component in the management of kidney cancer, however it poses a risk of perioperative complications including compromising renal function. ${ }^{1}$ To mitigate this, partial nephrectomy, when amenable, is recommended as it reduces the likelihood of developing chronic kidney disease (CKD) and cardiovascular morbidity and mortality. ${ }^{2-4}$ Population studies show that patients with concomitant kidney cancer and CKD have an increased risk of end stage renal disease (ESRD) and cardiovascular events. ${ }^{5,6}$ Dialysis-dependence increases mortality and reduces overall quality of life. ${ }^{7,8}$ Therefore, operating on patients with pre-existing CKD, balances the benefits of surgical intervention against the risk of further harming renal function.

We have previously validated factors predicting the restoration of baseline estimated glomerular filtration rate (eGFR) after radical nephrectomy. ${ }^{9,}{ }^{10}$ Patients with preoperative CKD $\left(\mathrm{eGFR}<60 \mathrm{~mL} / \mathrm{min} / 1.73 \mathrm{~m}^{2}\right.$ ) were more likely than those with normal renal function (eGFR $>60 \mathrm{~mL} / \mathrm{min} / 1.73 \mathrm{~m}^{2}$ ) to return to $95 \%$ of baseline eGFR within two years. For patients with preoperative CKD, eGFR recovery was associated with normotension and larger tumor size. However, we did not assess whether this recovery was transient or sustained, prevented longterm dialysis or improved survival.

Contemporary management of fragile patients involves multidisciplinary consideration of both surgical and non-surgical management strategies. ${ }^{11}$ In patients with concurrent CKD and kidney cancer, the decision to operate requires a careful assessment of both preoperatively appreciable tumor factors including size, vascular extension and clinical staging and the patient's fitness for surgery including age, renal function, comorbidities, tobacco use and body mass index (BMI). Herein, we aim to characterize the long-term renal function trajectory of patients with pre-existing CKD managed with surgery.

\section{Methods}

\section{Patient selection}

After obtaining Institutional Review Board approval, we queried our prospectively maintained kidney surgery database for patients with CKD who underwent a partial or radical nephrectomy between 1998-2016. We considered a patient to have CKD if their preoperative eGFR closest to surgery, calculated with the CKD-EPI equation, was $<60 \mathrm{~mL} / \mathrm{min} / 1.73 \mathrm{~m}^{2}{ }^{12}$

Patients rendered anephric after kidney tumor surgery $(n=6)$, on preoperative dialysis $(n=4)$, without an eGFR within six weeks of surgery $(n=4)$, or with incomplete baseline 
information $(n=5)$ were excluded, leaving a final study cohort of 1204 patients. To reduce bias from multiple procedures, patients receiving staged or repeat kidney tumor operations were followed from the time of their last procedure.

\section{Cohort variables}

Patient and disease characteristics were summarized with the median and interquartile range (IQR) for continuous variables and the frequency and percentage for binary and categorical variables. Patient and disease characteristics at baseline were compared across preoperative CKD stages using the Wilcoxon rank-sum test for continuous variables and the Chi-squared or Fisher's exact test, as appropriate, for binary and categorical variables. Cardiovascular risk factors, including diabetes, hypertension, and coronary artery disease, were recorded by the perioperative physician undertaking the preoperative patient physical evaluation. These factors were considered separately when describing patient characteristics and were combined into a single variable reflecting any cardiovascular risk factor in modelling to account for the common cooccurrence of these conditions.

Tumor stage is closely correlated to tumor size and, unlike size, accurate staging requires the pathological specimen. As we were assessing variables measurable preoperatively, tumor size and the presence of metastases were selected to evaluate tumor factors.

\section{Postoperative statistical analysis}

Our primary analysis was of postoperative longitudinal eGFR trajectories. First, we examined postoperative longitudinal trajectories of eGFR visually by plotting a smooth curve from a generalized additive model, grouping patients according to preoperative CKD stage. Univariable and multivariable mixed effects models were used to assess the association between preoperative patient and disease characteristics with longitudinal eGFR values. The mixed effects models incorporated a random intercept and slope for each patient, to allow for variation in both baseline eGFR and eGFR trajectory over time. The interaction effect between time from surgery and preoperative CKD stage with respect to eGFR over time was tested using a likelihood ratio test, and visually examined by plotting the predicted eGFR over time according to CKD stage for patients with baseline values of all other covariates. Patients initiating dialysis, either permanent or temporary, or receiving a kidney transplantation, were censored at the time of their intervention. Dialyzed patients were further investigated to determine their baseline characteristics, whether preoperative vascular access for dialysis was prepared, the indication for dialysis and whether a kidney transplant ensued.

Our secondary analysis was of survival outcomes for this population. Overall survival (OS) and cancer-specific survival (CSS) were defined as time from surgery to death from any cause or death from kidney cancer, respectively, and those still alive were censored at their last follow-up date. The Kaplan-Meier method estimated OS and CSS and the log-rank test was used to compare across preoperative CKD stages. Multivariable Cox regression was used to examine associations with OS and CSS. Time to dialysis was estimated using cumulative incidence, with death without dialysis treated as a competing event, and Gray's test was used to compare across 
preoperative $\mathrm{CKD}$ stages. A multivariable competing risks regression was used to test for associations with dialysis. Factors included in multivariable analysis were determined a priori based on clinical interest.

A p-value $<0.05$ was considered statistically significant. All statistical analyses were conducted using R version 3.5.1 (R Core Development Team, Vienna, Austria).

\section{Results}

There were 1204 patients included in our study cohort. The median age was 68 years (IQR: 6475 ) and $37 \%$ were women. The median time from measurement of preoperative eGFR to kidney surgery was 8 days (IQR: 5-13). At baseline, 892 (74\%) patients had stage 3a CKD, 271 (23\%) had stage $3 \mathrm{~b}$ and $41(3.4 \%)$ had stage 4 or $5(4 / 5)$ CKD. Patients with more severe CKD were older (median age 71 stage $3 \mathrm{~b}$ and $4 / 5$ versus 67 stage $3 \mathrm{a}$ ) and more frequently had hypertension ( $88 \%$ stage $4 / 5$ versus $79 \%$ stage 3 b versus $70 \%$ stage $3 a$ ) and coronary artery disease $(29 \%$ stage $4 / 5$ versus $24 \%$ stage 3 b versus $17 \%$ stage $3 a$ ). Tumor characteristics were similar across all groups (Table 1) with predominantly malignant pathology. (Supplementary Table 1)

Patients in all CKD groups more frequently had partial $(61 \%)$ rather than radical $(39 \%)$ nephrectomy. There were 25,781 postoperative eGFR measurements, with a median of 15 measurements per patient (IQR: 9-25). During the study period, 55 patients required dialysis and 355 patients died, 99 (28\%) of which were deaths due to kidney cancer. The median eGFR follow-up time among those alive without dialysis was 8.1 years (IQR: 4.6, 10). The raw eGFR measurements for each patient are plotted over time in Figure 1A, with patients grouped by preoperative CKD Stage and the generalized additive model smooth overlaid within each stage. The stage $3 b$ and stage $4 / 5$ CKD lines cross after 7 years, likely due to sparse data in the latter years.

For the primary analysis of postoperative eGFR trajectory, in multivariable mixed effects regression models incorporating a random intercept and slope as well as a fixed effect for time from kidney surgery, factors associated with increasing eGFR postoperatively included partial nephrectomy, female gender, younger age, lower BMI and the absence of any cardiovascular risk factors. (Table 2) Furthermore, we identified a significant interaction effect between time from kidney surgery and preoperative CKD stage (interaction $\mathrm{p}<0.001$ ). To demonstrate this interaction effect, we plotted a model predicting eGFR according to time from surgery for a patient with baseline levels of all other covariates, separately for each preoperative CKD stage (Figure 1B). This theoretical model illustrated that patients with stage $3 \mathrm{~b}$ or stages $4 / 5$ disease at baseline had decreased eGFR over time whereas patients with stage 3 a disease at baseline had an increased eGFR with time from surgery.

Dialysis was initiated in 55 patients, with a 2-year and 5-year cumulative incidence of dialysis of $1.8 \%$ (95\% confidence interval (CI): $1.1-2.6 \%$ ) and $3.1 \%$ (95\% CI: $2.2-4.2 \%$ ), respectively. The cumulative incidence of dialysis, with death as a competing event, significantly differed according to preoperative CKD stage (Figure 2). Patients receiving a partial nephrectomy had a lower cumulative incidence of dialysis, although this difference did not quite 
reach conventional levels for statistical significance $(\mathrm{p}=0.05)$. The individual sequelae for dialysis varied, including three patients ultimately undergoing kidney transplantation. Preoperative CKD stage was significantly associated with OS, but not CSS (Figure 3), such that those with stage G3b and G4/5 disease had a lower overall survival probability than those with stage G3a. On multivariable analysis, tumor factors including size and preoperative metastasis were significantly associated with OS and CSS, but not dialysis (Table 3). Consistent with other studies, female gender and increased BMI were associated with improved CSS. Increased age was associated with reduced OS, and interestingly, a reduced incidence of dialysis, likely due to the fact that older patients are dying of their comorbidities before they require dialysis for survival, as evidenced by the increased hazard of the competing risk of death with increasing age (hazard ratio: $1.04,95 \% \mathrm{CI}: 1.02-1.06, \mathrm{p}<0.001$ ).

\section{Discussion}

In this longitudinal study of renal function after kidney tumor surgery in patients with preoperative $\mathrm{CKD}$, after adjusting for other covariates, we found a divergence in the postoperative eGFR trajectory between patients with stage $3 \mathrm{a}$ CKD and those with stage $3 \mathrm{~b}$ or worse CKD. The patients with stage 3 a CKD increased their eGFR over time, above their baseline level, whereas the eGFR of patients with stage $3 \mathrm{~b}$ or worse CKD declined after kidney surgery. This preoperative distinction within the CKD population has not been described before.

Other factors associated with a reduced eGFR over time included increased BMI and the presence of cardiovascular diseases, including hypertension, diabetes and coronary artery disease. An elevated BMI is a potentially modifiable risk factor while diabetes and hypertension are also manageable chronic diseases. Although we have not tested the impact of managing these comorbidities on eGFR trajectory, our research reinforces the importance of screening for these medical conditions as they help predict the trajectory of renal function after surgery. Nonmodifiable risk factors for reduced eGFR over time included male gender and older age. These risk factors are also seen in kidney transplant donors who develop ESRD. ${ }^{13}$ Female gender was protective of eGFR in our cohort and previous studies. ${ }^{10,14}$ A study comparing survival of patients with ESRD from a trauma nephrectomy, oncological nephrectomy, or diabetes found that diabetics had greater non-cancer mortality. ${ }^{15}$ This reinforces the vulnerability of patients with concomitant medical comorbidities, CKD and kidney cancer.

A radical nephrectomy was associated with a reduced eGFR over time as well as worse CSS and OS. While this may be influenced by tumor stage, we adjusted for tumor size and the presence of metastases to mitigate this. Given all patients had residual nephrons after surgery, the reduced eGFR following radical nephrectomy may reflect a greater reduction in renal volume. Increased tumor size was significantly associated with a reduced eGFR over time on univariable analysis $(\mathrm{p}<0.001)$, but the direction of this effect reversed on multivariable analysis whereby increased tumor size was associated with an increased eGFR over time $(p=0.101)$. Other studies also observed that resecting larger tumors is associated with an improved eGFR postoperatively. ${ }^{10,16}$ This phenomenon may be explained by enlarging tumors gradually 
destructing healthy kidney prior to surgery, leading to compensatory upregulation of the contralateral kidney to maintain homeostasis. This effect is likely to be less pronounced in smaller tumors. Importantly, the bidirectional difference in eGFR trajectory between stage 3a CKD and stage 3b-5 CKD was observed after both radical nephrectomy and partial nephrectomy. As patients with CKD are vulnerable to worsening eGFR and all-cause mortality, performing a partial nephrectomy, if it is technically feasible, should be considered when protecting renal function is a key concern.

Preoperative CKD stage was significantly associated with the risk of dialysis. A previous study also reported that an increasing preoperative eGFR and partial nephrectomy protected against dialysis. ${ }^{17}$ Another study described partial nephrectomy in 84 patients with solitary kidneys, reporting an increased risk of dialysis with lower preoperative eGFR. In the study, only patients with preoperative CKD developed ESRD, with a five year ESRD-free survival rate of 97\%. ${ }^{18}$ In our cohort, most patients requiring dialysis commenced during outpatient follow-up, including patients that subsequently underwent a kidney transplant. Partial nephrectomy was marginal for reducing the cumulative incidence of dialysis $(p=0.05)$. Notably, only stage $3 a$ and $3 \mathrm{~b}$ patients were weaned off inpatient dialysis. Almost all patients requiring inpatient dialysis received a preoperative dialysis catheter or arteriovenous fistula. Overall, the 5-year cumulative dialysis incidence was $3.1 \%$.

This study has limitations since it is single institutional and retrospective. Generalizability is limited by institutional variations in patient selection, the management of comorbidities, and policies regarding dialysis and transplantation. Reassuringly, significant variables in our cohort, other than CKD stage, are consistent with a similar longitudinal study in mostly healthy kidneys looking at predictors of eGFR post partial and radical nephrectomy. ${ }^{19}$ The strengths of this data are the large sample size, long-term detailed follow up and depth of patient-level information with detailed insight into medical comorbidities, repeat procedures and the status of residual kidney tissue. Important preoperative considerations not studied here which can also provide insight include RENAL Nephrometry scores and split renal function tests. Population-based trials screen for events representative of ESRD, ${ }^{14,20}$ however they tend not to incorporate the longitudinal tracking of eGFR trends. These advantages have enabled this study to carefully determine the long-term postoperative patterns and drivers of eGFR changes and dialysis rates therein.

\section{Conclusions}

When considering patients with CKD for kidney surgery, our research indicates that preoperative CKD at stage $3 \mathrm{~b}$ or worse is associated with a higher risk of further reduction in eGFR, the need for dialysis, and increased all-cause mortality for both partial and radical nephrectomy.

Nonetheless, among a large cohort of patients with preoperative CKD, carefully selected patients can be managed through surgery with a low rate of eventual dialysis. 
Future research to improve outcomes should focus on early interventions that optimize renal function and treat modifiable comorbidities, for both surgical and non-surgical management strategies.

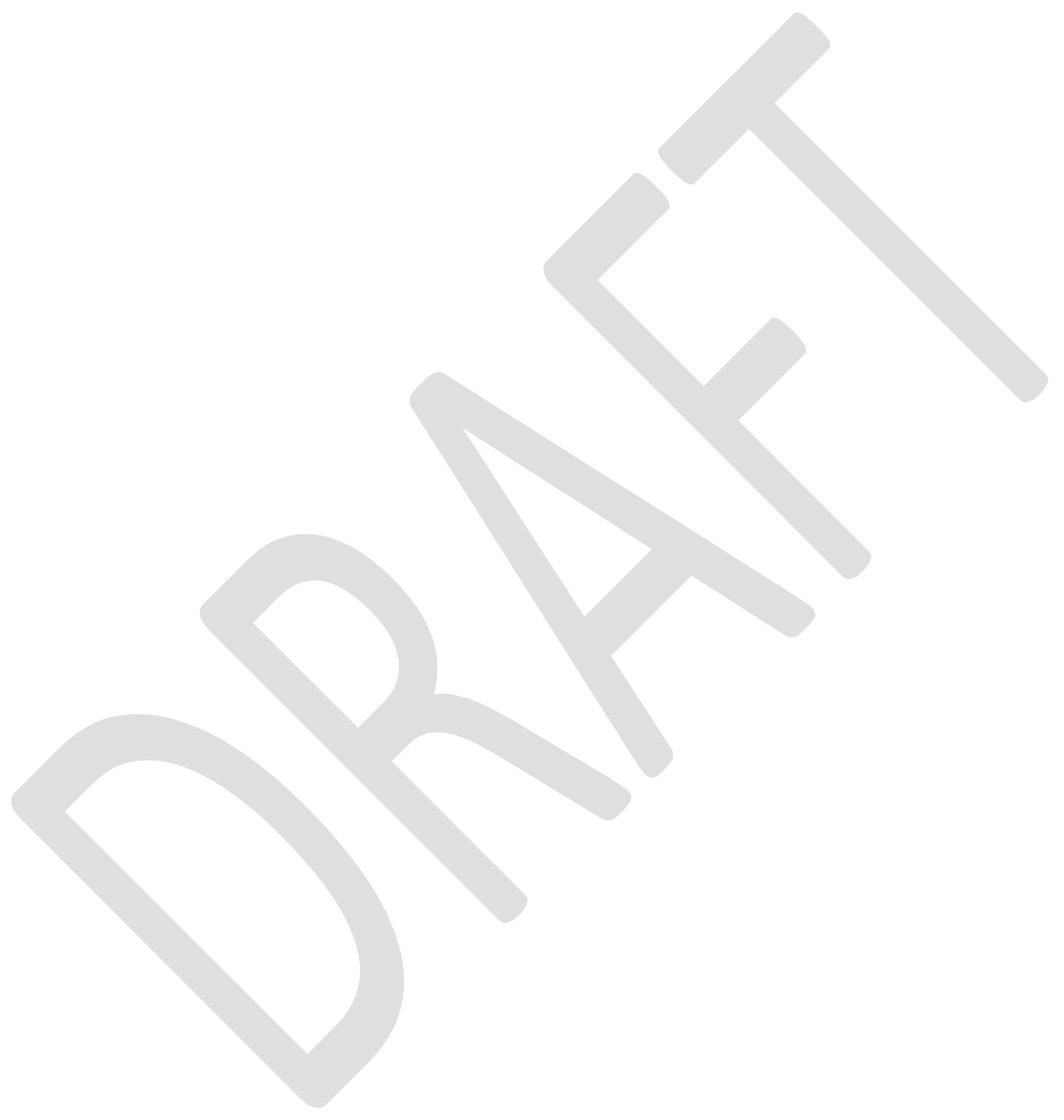

\section{References}


1. Scosyrev E, Messing EM, Sylvester R, Campbell S, Van Poppel H. Renal function after nephron-sparing surgery versus radical nephrectomy: results from EORTC randomized trial 30904. European urology. 2014;65:372-377.

2. Mir MC, Derweesh I, Porpiglia F, Zargar H, Mottrie A, Autorino R. Partial Nephrectomy Versus Radical Nephrectomy for Clinical T1b and T2 Renal Tumors: A Systematic Review and Meta-analysis of Comparative Studies. European urology. 2017;71:606-617.

3. Kaushik D, Kim SP, Childs MA, et al. Overall survival and development of stage IV chronic kidney disease in patients undergoing partial and radical nephrectomy for benign renal tumors. European urology. 2013;64:600-606.

4. Leppert JT, Lamberts RW, Thomas IC, et al. Incident CKD after Radical or Partial Nephrectomy. Journal of the American Society of Nephrology : JASN. 2018;29:207216.

5. Weight CJ, Larson BT, Fergany AF, et al. Nephrectomy induced chronic renal insufficiency is associated with increased risk of cardiovascular death and death from any cause in patients with localized cT1b renal masses. The Journal of urology. 2010;183:1317-1323.

6. Weiner DE, Tighiouart H, Amin MG, et al. Chronic kidney disease as a risk factor for cardiovascular disease and all-cause mortality: a pooled analysis of community-based studies. Journal of the American Society of Nephrology : JASN. 2004;15:1307-1315.

7. Neovius M, Jacobson SH, Eriksson JK, Elinder CG, Hylander B. Mortality in chronic kidney disease and renal replacement therapy: a population-based cohort study. BMJ open. 2014;4:e004251.

8. Mazairac AH, de Wit GA, Penne EL, et al. Changes in quality of life over time-Dutch haemodialysis patients and general population compared. Nephrology, dialysis, transplantation : official publication of the European Dialysis and Transplant Association - European Renal Association. 2011;26:1984-1989.

9. Zabor EC, Furberg H, Mashni J, Lee B, Jaimes EA, Russo P. Factors Associated with Recovery of Renal Function following Radical Nephrectomy for Kidney Neoplasms. Clinical journal of the American Society of Nephrology : CJASN. 2016;11:101-107.

10. Zabor EC, Furberg H, Lee B, et al. Long-Term Renal Function Recovery following Radical Nephrectomy for Kidney Cancer: Results from a Multicenter Confirmatory Study. The Journal of urology. 2018;199:921-926.

11. Campbell S, Uzzo RG, Allaf ME, et al. Renal Mass and Localized Renal Cancer: AUA Guideline. The Journal of urology. 2017;198:520-529.

12. Levey AS, Stevens LA, Schmid CH, et al. A new equation to estimate glomerular filtration rate. Annals of internal medicine. 2009;150:604-612.

13. Massie AB, Muzaale AD, Luo X, et al. Quantifying Postdonation Risk of ESRD in Living Kidney Donors. Journal of the American Society of Nephrology : JASN. 2017;28:2749-2755.

14. Ellis RJ, Edey DP, Del Vecchio SJ, et al. End-Stage Kidney Disease following Surgical Management of Kidney Cancer. Clinical journal of the American Society of Nephrology : CJASN. 2018;13:1641-1648.

15. Nguyen KA, Vourganti S, Syed JS, Luciano R, Campbell SC, Shuch B. End-stage renal disease secondary to renal malignancy: Epidemiologic trends and survival outcomes. Urologic oncology. 2017;35:529.e521-529.e527. 
16. Ohno Y, Nakashima J, Ohori M, et al. Impact of tumor size on renal function and prediction of renal insufficiency after radical nephrectomy in patients with renal cell carcinoma. The Journal of urology. 2011;186:1242-1246.

17. Takagi T, Kondo T, Omae K, et al. Comparison of progression to end-stage renal disease requiring dialysis after partial or radical nephrectomy for renal cell carcinoma in patients with severe chronic kidney disease. Int Urol Nephrol. 2016;48:1421-1427.

18. La Rochelle J, Shuch B, Riggs S, et al. Functional and oncological outcomes of partial nephrectomy of solitary kidneys. The Journal of urology. 2009;181:20372042; discussion 2043.

19. Bhindi B, Lohse CM, Schulte PJ, et al. Predicting Renal Function Outcomes After Partial and Radical Nephrectomy. European Urology. 2019;75:766-772.

20. Lin WY, Liang FW, Lu TH. Risk of end-stage renal disease after cancer nephrectomy in Taiwan: a nationwide population-based study. PloS one. 2015;10:e0126965. 


\section{Figures and Tables}

Fig. 1. (A) Longitudinal estimated glomerular filtration rate (eGFR) over time with smooth based on general additive model. (B) Interaction between years since surgery and preoperative chronic kidney disease (CKD) stage from the multivariable mixed effects model, for a patient with baseline levels of all other covariates.

A

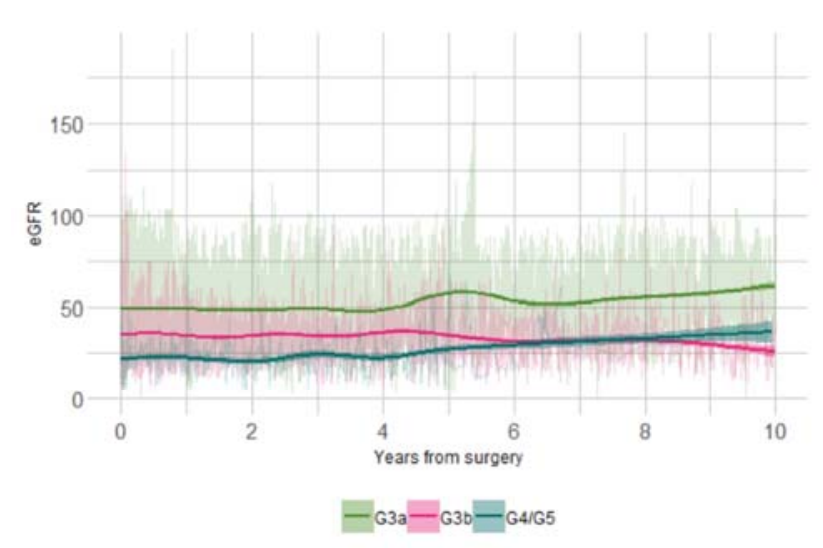

B

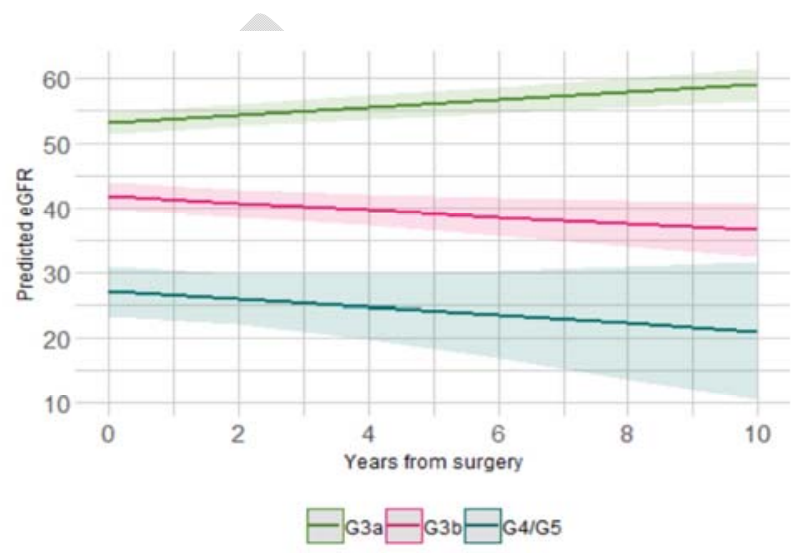

Fig. 2. (A) Plot of time to dialysis, where death is treated as a competing event. (B) Heatmap description of patients requiring dialysis.

A

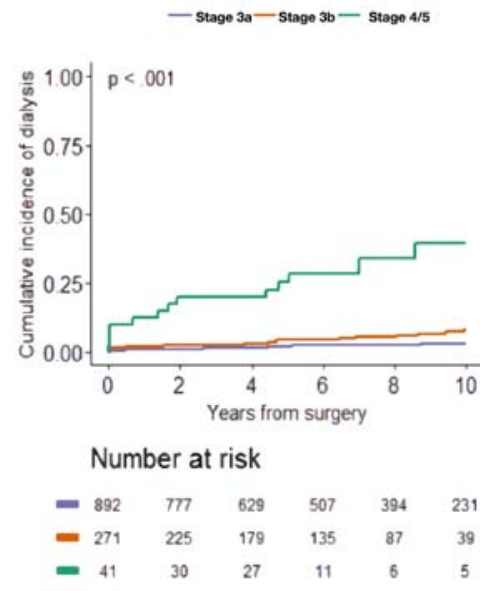

B

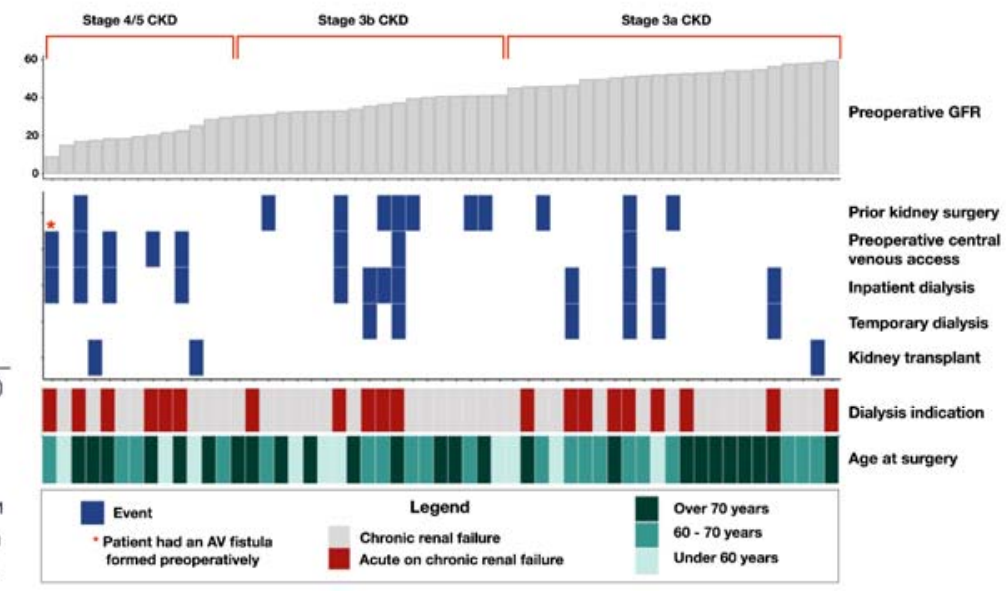


Fig. 3. Plots of (A) cancer-free survival; and (B) overall survival according to preoperative chronic kidney disease (CKD) stage.

A

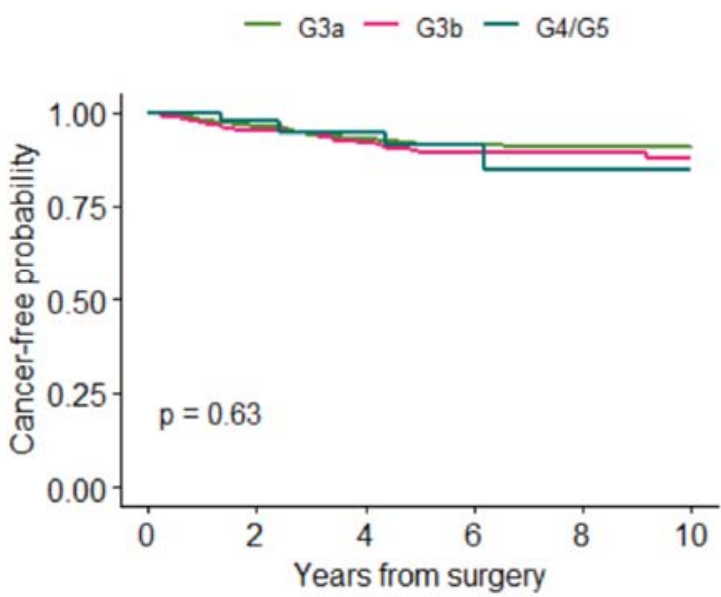

Number at risk

$\begin{array}{rrrrrr}892 & 780 & 633 & 514 & 397 & 233 \\ -271 & 231 & 184 & 140 & 91 & 46 \\ -\quad 41 & 36 & 28 & 14 & 8 & 6\end{array}$

B

$-\mathrm{G}_{\mathrm{a}} \mathrm{-}-\mathrm{G} 3 \mathrm{~b}-\mathrm{G} 4 / \mathrm{G} 5$

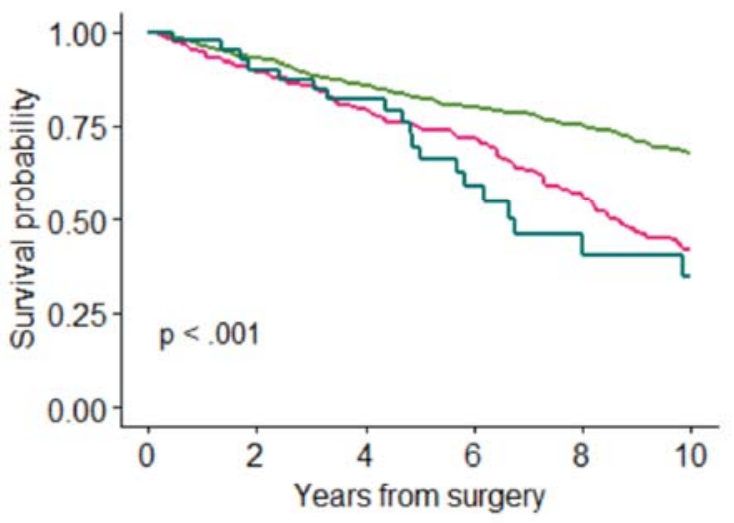

Number at risk

$\begin{array}{rrrrrr}892 & 780 & 633 & 514 & 397 & 233 \\ =271 & 231 & 184 & 140 & 91 & 46 \\ =41 & 36 & 28 & 14 & 8 & 6\end{array}$

\begin{tabular}{|l|c|c|c|c|}
\hline \multicolumn{1}{|l|}{ Table 1. Preoperative patient characteristics by CKD stage } \\
\hline Variable & G3a & G3b & G4/G5 & p \\
\hline & $\mathbf{n = 8 9 2 ( 7 4 \% )}$ & $\mathbf{n = 2 7 1}(\mathbf{2 3} \%)$ & $\mathbf{n = 4 1} \mathbf{( 3 . 4 \% )}$ & \\
\hline Female sex & $328(37 \%)$ & $109(40 \%)$ & $12(29 \%)$ & 0.3 \\
\hline White race & $827(93 \%)$ & $251(93 \%)$ & $36(88 \%)$ & 0.5 \\
\hline Age at surgery (years) & $67(60,73)$ & $71(65,77)$ & $71(64,75)$ & $<0.001$ \\
\hline BMI & $29.1(26.0,32.5)$ & $28.4(25.5,32.4)$ & $29.4(25.7,32.5)$ & 0.4 \\
\hline \multicolumn{1}{|c|}{ Unknown BMI } & 75 & 25 & 4 & 0.11 \\
\hline Ever Smoker & $430(48 \%)$ & $145(54 \%)$ & $25(61 \%)$ & 0.5 \\
\hline Preoperative metastases & $73(8.2 \%)$ & $21(7.7 \%)$ & $1(2.4 \%)$ & 0.4 \\
\hline Preoperative diabetes & $147(16 \%)$ & $52(19 \%)$ & $9(22 \%)$ & 0.001 \\
\hline Preoperative hypertension & $623(70 \%)$ & $214(79 \%)$ & $36(88 \%)$ & 0.009 \\
\hline $\begin{array}{l}\text { Preoperative coronary } \\
\text { artery disease }\end{array}$ & $152(17 \%)$ & $65(24 \%)$ & $12(29 \%)$ & 0.3 \\
\hline Radical nephrectomy & $350(39 \%)$ & $113(42 \%)$ & $12(29 \%)$ & 0.11 \\
\hline Tumor size (cm) & $3.8(2.5,6.2)$ & $4.2(2.5,7.0)$ & $4.5(3.2,7.0)$ & \\
\hline
\end{tabular}

BMI: body mass index. CKD: chronic kidney disease. 
Long-term renal function after kidney surgery in CKD patients

\begin{tabular}{|l|c|c|c|}
\hline \multicolumn{3}{|l|}{ Table 2. Multivariable mixed effects model for factors associated with eGFR over time } \\
\hline Variable & Estimate & Standard error & p \\
\hline Years from procedure & 0.580 & 0.105 & $<0.001$ \\
\hline Gender (female) & 4.53 & 0.643 & $<0.001$ \\
\hline Age & -0.293 & 0.034 & $<0.001$ \\
\hline Race (non-White) & -1.53 & 1.16 & 0.2 \\
\hline BMI & -0.199 & 0.059 & $<0.001$ \\
\hline Smoking history & -0.266 & 0.615 & 0.7 \\
\hline Preoperative metastases & 1.94 & 1.16 & 0.09 \\
\hline Any cardiovascular risk factors & -2.41 & 0.762 & 0.002 \\
\hline Radical nephrectomy & -9.96 & 0.776 & $<0.001$ \\
\hline Tumor size & 0.187 & 0.113 & $<0.001$ \\
\hline Preoperative CKD stage & & & \\
\hline G3a & Ref & & \\
\hline G3b & -11.2 & & \\
\hline G4/G5 & -25.9 & & \\
\hline Years from procedure x preoperative CKD & & & \\
\hline $\begin{array}{l}\text { Years from procedure x preoperative CKD } \\
\text { G3a }\end{array}$ & Ref & & \\
\hline $\begin{array}{l}\text { Years from procedure x preoperative CKD } \\
\text { G3b }\end{array}$ & -1.11 & & \\
\hline $\begin{array}{l}\text { Years from procedure x preoperative CKD } \\
\text { G4/G5 }\end{array}$ & -1.20 & 0.776 & \\
\hline BMI: body mass index; CKD: & & \\
\hline
\end{tabular}

BMI: body mass index; CKD: chronic kidney disease; eGFR: estimated glomerular filtration rate. 


\begin{tabular}{|c|c|c|c|c|c|c|}
\hline & $\begin{array}{l}\text { Dialysis HR } \\
(95 \% \mathrm{CI}) \\
\end{array}$ & $\begin{array}{c}\text { Dialysis } \\
\mathbf{p} \\
\end{array}$ & $\begin{array}{c}\text { CSS HR } \\
(95 \% \text { CI }) \\
\end{array}$ & $\begin{array}{c}\text { CSS } \\
\mathbf{p} \\
\end{array}$ & $\begin{array}{c}\text { OS HR } \\
(95 \% \mathrm{CI}) \\
\end{array}$ & $\begin{array}{c}\mathbf{O S} \\
\mathbf{p} \\
\end{array}$ \\
\hline Gender (female) & $0.77(0.38,1.59)$ & 0.5 & $0.60(0.37,0.96)$ & 0.03 & $0.85(0.66,1.08)$ & 0.2 \\
\hline Age & $0.97(0.94,1.00)$ & 0.02 & $1.00(0.98,1.03)$ & 0.8 & $1.04(1.02,1.05)$ & $<0.001$ \\
\hline Race (non-White) & $1.18(0.39,3.56)$ & 0.8 & $0.59(0.25,1.39)$ & 0.2 & $0.94(0.61,1.44)$ & 0.8 \\
\hline BMI & $1.01(0.97,1.06)$ & 0.6 & $0.94(0.90,0.98)$ & 0.005 & $0.99(0.97,1.01)$ & 0.3 \\
\hline Smoking history & $1.51(0.77,2.96)$ & 0.2 & $0.71(0.46,1.10)$ & 0.13 & $1.07(0.85,1.34)$ & 0.6 \\
\hline $\begin{array}{l}\text { Preoperative } \\
\text { metastases }\end{array}$ & $1.71(0.54,5.38)$ & 0.4 & $7.44(4.75,11.7)$ & $<0.001$ & $4.02(2.99,5.41)$ & $<0.001$ \\
\hline $\begin{array}{l}\text { Any cardiovascular } \\
\text { risk factors }\end{array}$ & $1.78(0.69,4.60)$ & 0.2 & $0.81(0.51,1.30)$ & 0.4 & $0.92(0.70,1.20)$ & 0.5 \\
\hline Radical nephrectomy & $1.88(0.96,3.70)$ & 0.07 & $2.51(1.36,4.63)$ & 0.003 & $1.33(1.01,1.76)$ & 0.04 \\
\hline Tumor size & $0.97(0.87,1.08)$ & 0.5 & $1.13(1.07,1.19)$ & $<0.001$ & $1.08(1.04,1.11)$ & $<0.001$ \\
\hline $\begin{array}{l}\text { Preoperative CKD } \\
\text { stage }\end{array}$ & & $<0.001$ & & 0.6 & & $<0.001$ \\
\hline G3a & Ref & & Ref & & Ref & \\
\hline $\mathrm{G} 3 \mathrm{~b}$ & $3.23(1.52,6.86)$ & 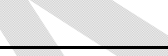 & $1.18(0.73,1.91)$ & 0.5 & $1.55(1.22,1.99)$ & \\
\hline G4/G5 & $16.6(6.76,40.8)$ & & $1.57(0.55,4.43)$ & 0.4 & $1.94(1.17,3.23)$ & \\
\hline
\end{tabular}

BMI: body mass index; CKD: chronic kidney disease; CI: confidence interval; CSS: cancer-specific survival; HR: hazard ratio; OS: overall survival. 
Long-term renal function after kidney surgery in CKD patients

\begin{tabular}{|c|c|}
\hline \multicolumn{2}{|c|}{ Supplementary Table 1. Tumor characteristics } \\
\hline Variable & $\mathrm{n}=1204$ \\
\hline Tumor size & $4.0(2.5,6.5)$ \\
\hline \multicolumn{2}{|l|}{ T stage } \\
\hline $\mathrm{TX}$ & $8(0.7 \%)$ \\
\hline T0 & $69(5.8 \%)$ \\
\hline $\mathrm{T} 1$ & $681(57 \%)$ \\
\hline $\mathrm{T} 2$ & $73(6.1 \%)$ \\
\hline $\mathrm{T} 3$ & $354(30 \%)$ \\
\hline $\mathrm{T} 4$ & $12(1.0 \%)$ \\
\hline Unknown & 7 \\
\hline \multicolumn{2}{|l|}{ N stage } \\
\hline N0 & $374(34 \%)$ \\
\hline N1 & $19(1.7 \%)$ \\
\hline $\mathrm{N} 2$ & $22(2.0 \%)$ \\
\hline NX & $684(62 \%)$ \\
\hline Unknown & 105 \\
\hline \multicolumn{2}{|l|}{ Met status } \\
\hline M0 & $1005(83 \%)$ \\
\hline M1 & $199(17 \%)$ \\
\hline \multicolumn{2}{|l|}{ Margins } \\
\hline Negative & $1089(92 \%)$ \\
\hline Close & $6(0.5 \%)$ \\
\hline Positive & $90(7.6 \%)$ \\
\hline Unknown & 19 \\
\hline \multicolumn{2}{|l|}{ Histology } \\
\hline Clear-cell RCC & $677(56 \%)$ \\
\hline Non-clear-cell RCC & $450(38 \%)$ \\
\hline Other (non-RCC) & $72(6.0 \%)$ \\
\hline Unknown & 5 \\
\hline
\end{tabular}

RCC: renal cell carcinoma. 